\title{
Review of: "Structural analysis of Red1 as a conserved scaffold of the RNA-targeting MTREC/PAXT complex"
}

Mathieu Rougemaille

Potential competing interests: The author(s) declared that no potential competing interests exist.

In this study, Foucher and coworkers carry out a structure-based analysis of the MTREC RNA-targeting complex in fission yeast, involved in the degradation of various RNA species including meiotic mRNAs and CUTs. The authors combine biochemical, structural and in vivo approaches to show that i) the MTREC subunit Red1 directly associates with the Iss10 and Ars2 components using distinct interaction regions, ii) point mutations in Red1 disrupting its association with Iss10 and Ars2 impact cell growth, protein localization and/or RNA degradation, iii) the Red1-Ars2 interaction is conserved in human homologs (hZFC3H1/hARS2), and iv) Red1 has the ability to dimerize.

Overall, this is an interesting study revealing structural bases for the association of different MTREC subunits to the core component Red1 and the data are overall of high quality. Although the Red1-Iss10 and Red1-Ars2 interactions reported in this manuscript were recently described in an independent work (PMID 34103492), the authors further provide structural data, giving important molecular details about MTREC assembly, as well as evidence for Red1 dimerization, which is novel and intriguing.

Below are comments that might help improve the manuscript:

1) Figure 3a: since Iss10 total protein levels decrease in the Red1-L205-TAP mutant, the authors could load diluted samples (SE and IP) of the Red1-TAP Iss10-GFP strain to ease comparison with samples of the Red1L205-TAP Iss10-GFP strain. This is important because it seems that there is residual Iss10-GFP signal upon Red1-L205-TAP pulldown (last two lanes).

2) Figure 3d: The fact that Red1-L205-TAP forms additional nuclear foci is striking. Does an Iss10 Nterminal mutant result in the same phenotype?

3) Figures 5g: The Red1-Ars2 interaction is rather weak. Could the authors increase the starting material (i.e. more than $20 \mathrm{~mL}$ cultures) to better visualize the association?

4) Figure 5i: The authors state that the Ars2 localization is more diffuse in the Red1-E32R mutant but the 
data are not that clear. Would it be possible to provide some kind of quantification for "nuclear foci" versus "diffuse" signal?

5) Supplementary Figure 8b,c: The Red1-E32R mutation does not impact the levels of meiotic mRNAs and CUTS/PROMPTs, yet it affects cell fitness on minimal medium as red1 $\Delta$ cells do. Hence, does the E32R mutation impair the steady state levels of other Red1-targeted transcripts, including the prt and nam1 IncRNAs that lie upstream of the pho1+ and byr2+ genes, respectively (PMID32415063)?

6) Figure 7: The data supporting Red1 dimerization are really interesting. In line with the Red1-Iss10 and Red1-Ars2 interactions, a functional analysis of the Red1-L481R,1485R mutant (i.e. growth assay, localization, in vivo self-association and RNA degradation) would definitely strengthen the manuscript. Does the L481R, I485R mutant show a growth phenotype in minimal medium? Does it form additional nuclear foci, similar to the E32R mutant, or instead preclude their formation? Does it affect the levels of meiotic mRNAs, IncRNAs and/or CUTs?

7) Figure 7f/Model: As mentioned in the discussion, Mmi1 forms an heterotetrameric complex with Erh1 (i.e. EMC), whereby an Erh1 homodimer bridges two Mmi1 molecules (PMID30651569; PMID31974447). Hence, an attractive possibility could be that Red1 dimers directly connect Iss10-bound EMC (i.e. Red1Iss10-Mmi1-Erh1-Erh1-Mmi1-Iss10-Red1). The authors could discuss this point and highlight its potential implications.

Minor points:

1) Page 6, paragraph "The Red1-Iss10 interface": “...including contacts between Iss10 Ala198, Ala201 and Leu205 and Red1 Ile15 and Phe18" should be “...including contacts between Red1 Ala198, Ala201 and Leu205 and Iss10 Ile15 and Phe18".

2) Supplementary Figure 8c: on the graph, it should be written rlp402 "PROMPT", not PROMT. 